\title{
Prevalence of Tinea Corporis and Tinea Cruris in Outpatient Department of Dermatology Unit of a Tertiary Care Hospital
}

\author{
Brigida $S^{*}$ and Muthiah NS \\ Department of Pharmacology, Sree Balaji Medical College and Research Hospital, India
}

Submission: March 06, 2017; Published: May 30, 2017

*Corresponding author: Brigida S, Department of Pharmacology, Sree Balaji Medical College and Research Hospital, Chrompet, ChennaiTamilnadu, India, E-mail: 1506dr.brigida@gmail.com

\begin{abstract}
Background: Dermatophytes are the major causal organism of the most common superficial fungal infections. Among that Tinea corporis and Tinea cruris infections are not uncommon among general population irrespective of their glycemic or hygienic status.

Objective: This study is to evaluate the prevalence of Tinea corporis and Tinea cruris among different age groups in outpatient department of dermatology unit of Sree Balaji medical college and research hospital.

Result: In this present study out of 587 patients attended skin OPD with skin lesion or complaints, dermatologist diagnosed as 151 patients had Tinea corporis infection and 138 had Tinea cruris infection. Out of 151 Tinea corporis patients 84 are male and 67 are female. In male 75 patients are $\mathrm{KOH}$ positive and remaining are negative. In female 62 are $\mathrm{KOH}$ positive and 5 are $\mathrm{KOH}$ negative. Total of 138 Tinea cruris patient reported out of which 62 are males and 76 are females. Out of 62 in males 59 and out of 76 in females 73 are KOH positive and 3 in male and 3 in female are $\mathrm{KOH}$ negative. It is found that higher incidence of Tinea corporis in male and Tinea cruris in female.
\end{abstract}

Keywords: Prevalence, Superficial Fungal infection, KOH mount, microscopic examination

\section{Introduction}

Dermatophytes are filamentous fungi in the genera Trichophyton, Microsporum and Epidermophyton of which Trichophyton is the common species responsible for Tinea infections. More than a million healthy as well as immuno compromised are the victims of this infection worldwide. Dermatophytes manifest in humans in different forms and most common major infections are Tinea corporis and Tinea cruris most commonly caused by Trichophyton species, that disrupts keratin in the stratum corneum (epidermis of skin). This is because the dermatophyte group of fungi are unable to penetrate tissues which are not fully keratinized. However its severity varies from mild to moderate according to host immunity, virulence of the infecting species, site of infection and environmental factors [1-5].

Tinea corporis, one of the superficial dermatophyte infection manifest either as an inflammatory or non inflammatory lesions on the glabrous skin, most commonly trunk, shoulder and limbs (except scalp, groin, palms and soles)1. It is most commonly caused by Trichophyton species that digest keratin in the cells of the stratum corneum. T. rubrum is the most common infectious agent in the world and is the source of $47 \%$ of Tinea corporis cases1.It starts as a flat, scaly and more often as a pruritic macules which may further develop into a lesion with raised border spreading radially with erythematous vesicular edges. This may expand as a ring with central clearance and irregular circles giving this infection a layman name ring worm. Tinea corporis occurs worldwide and is relatively frequent, but its incidence is higher in tropics and subtropics. Infection can occur from direct or indirect contact with skin and scalp lesions of infected persons or animals.

Tinea cruris, the other variety of superficial Trichophyton infection, mostcommonly caused by T.rubrum, E. floccosum which manifest as an inflammatory lesion or asymptomatic,(depends on species virulence and patient's immune response) most 
commonly in the groin and genital area(perianal and perineal sites) with a tendency to use keratin as its nitrogen source for growth. Lesions are erythematous and covered with thin, dry scales, can extend to inner thigh with raised, defined border with small vesicles [6-8].

\section{Aim and Objective}

In this study our aim was to evaluate the prevalence of Tinea corporis and Tinea cruris among different age group in outpatient department of dermatology unit of Sree Balaji medical college and Research Hospital.

\section{Materials and Methods}

The present study has been conducted on a symptomatic group who visited dermatological OPD of Sree Balaji Medical college and research hospital.

\section{Selection of Patients}

Patients of all age group who visited OPD with complaint of itching or lesion were screened and then filtered after the diagnosis by dermatologist as Tinea corporis and Tinea cruris were included in the study irrespective of their comorbid condition. A brief history about any systemic diseases was noted. Most patients with tinea corporis and tinea cruris are diagnosed clinically. To avoid a misdiagnosis, identification of dermatophyte infections from skin scraping is suggestible. And hence the patient was taken to mycological unit of Microbiology department. Microscopic examination, consisting of a $10 \%$ to $15 \% \mathrm{KOH}$ preparation, from skin scrapings from peripheral margins and roof of the blister using small scalpel blade, dermatophytes are easily recognized under microscope by their long branch like tubular structures called HYPHAE and it was taken as confirmation.

\section{Study Period}

This study was conducted for a period of one year from September 2015-August 2016 after approval from institutional ethics committee.

\section{Results}

In this present study out of 587 patients attended skin OPD with skin lesion or complaints, dermatologist diagnosed as 151 patients had Tinea corporis infection and 138 had Tinea cruris infection (Figure 1). The initial diagnosis was done by dermatologist and after that mycological confirmation of presence of hyphae in $\mathrm{KOH}$ mounts under microscopy (Figure 2). The graph shows 31-45 years age group have greater incidence of Tinea corporis infection with male predominance. Out of 151 Tinea corporis patients 84 are male and 67 are female (Figure 3). In male 75 patients are $\mathrm{KOH}$ positive and remaining are negative. In female 62 are $\mathrm{KOH}$ positive and 5 are $\mathrm{KOH}$ negative (Figure 4). The graph shows16-45 years age group have greater incidence of Tinea cruris infection with female predominance. Total of 138 Tinea cruris patient reported out of which 62 are males and 76 are females (Figure 5). Out of 62 in males 59 and out of 76 in females 73 are $\mathrm{KOH}$ positive and 3 in male and 3 in female are $\mathrm{KOH}$ negative.

\section{TOTAL NUMBER OF TINEA CORPORIS AND CRURIS}

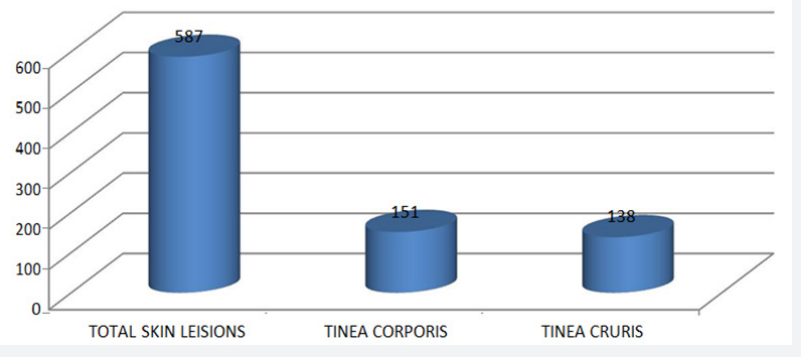

Figure 1: Total Tinea Corporis and Cruris Cases.

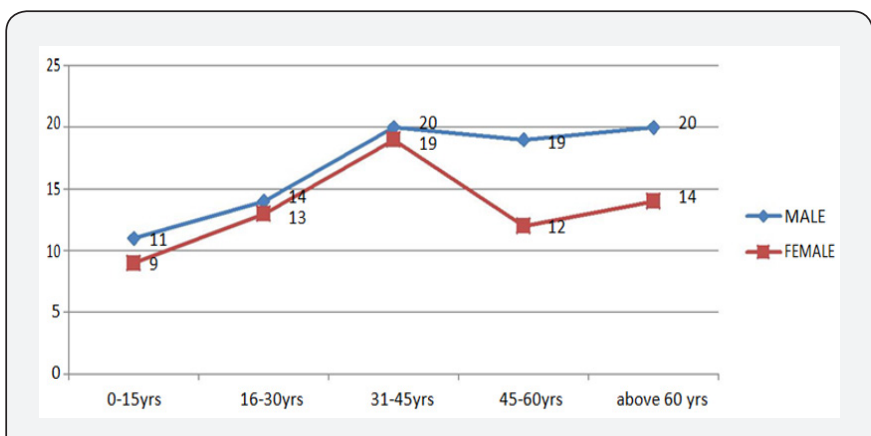

Figure 2: Male and Female Distribution of Tinea Corporis.

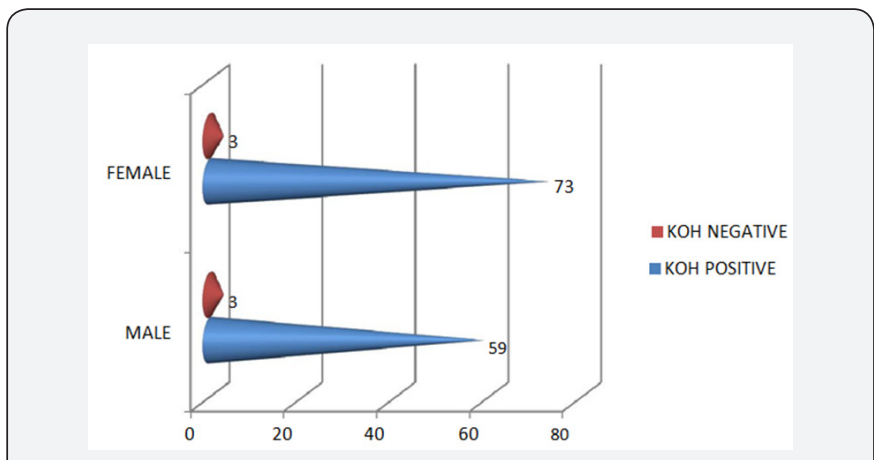

Figure 3: $\mathrm{KOH}$ Positive and $\mathrm{KOH}$ Negative Cases.

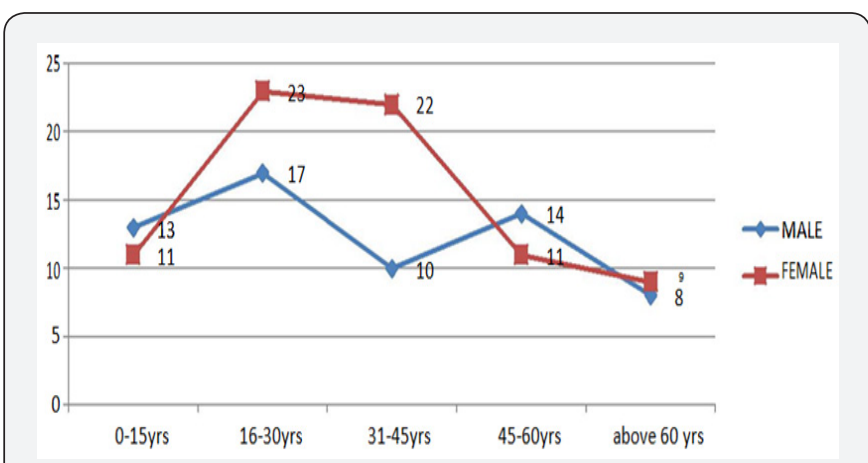

Figure 4: Male and Female Distribution of Tinea Cruris. 


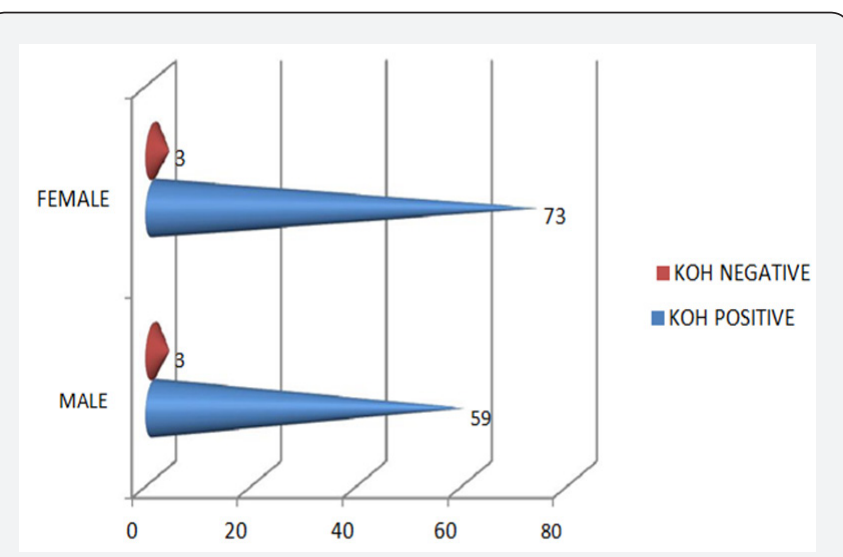

Figure 5: $\mathrm{KOH}$ Positive and $\mathrm{KOH}$ Negative Tinea Cruris Cases.

\section{Discussion}

In this era, most infections either of bacterial or fungal origin can be diagnosed properly with specific pertained investigations and due to development of several drugs with lesser adverse effects. In developing countries like India, Fungal infections assumed very great importance because of poor hygiene and sanitation awareness and because of climate in tropical region like Chennai. Although most superficial fungal infections are not life threatening, it may turn fatal due to systemic invasion in immunocompromised individuals or as an opportunistic infection during debilitation. Or even can have debilitating effects on an individual's quality of life $[9,10]$.

\section{Conclusion}

It may be concluded that in tropical countries like India dermatophyte infections like Tinea corporis and Tinea cruris are not uncommon irrespective of rural or urban population or irrespective of patient's co morbid conditions or hygienic status. As most of the skin lesions will not recur if it is addressed completely by targeting the pathology systemically and topically as well as by proper hygienic measures. Patients need to be educated regarding the continuation of medications until mycological cure in order to prevent recurrence and also about the hygienic measures.

\section{Limitations}

Further detailed study can be conducted to analyze the risk factors for Tinea corporis and Tinea cruris infection. In this study $\mathrm{KOH}$ negative cases were excluded and they can be further evaluated through culture to confirm the diagnosis and it might show variation in prevalence. Similarly detailed history about the recurrence of infection if any can help us to find the contribution of underlying co morbidities like diabetes.

\section{Acknowledgement}

My sincere thanks to Department of dermatology and department of Microbiology. My heartfelt gratitude to my beloved professors, faculty, peers and well wishers.

\section{References}

1. Gupta Sarika, Agrawal Purva, Rajawat Rahul, Gupta Saksham (2014) Prevalence Of Dermatophytic Infection And Determining Sensitivity Of Diagnostic Procedures. International Journal of Pharmacy and Pharmaceutical Sciences 6(3).

2. Svejgaard E (1986) Epidemiology and clinical features of dermatomycoses and dermatophytoses. Acta Derm Venereo Suppl (Stockh)121: 19-26.

3. Tan H H (2005) Superficial fungal infections seen at the National Skin Centre. Singapore. Jpn J Med Mycol 46(2): 77-80.

4. Andrews M D, Burns M (2008) Common Tinea infections in children. Am Fam Physician 77(10): 1415-1420.

5. Garg J, Tilak R, Garg A, Prakash P, Gulati A K, et al. (2009) Rapid detection of dermatophytes from skin and hair. BMC Res Notes 18: 2: 60.

6. ALY R (1994) Ecology and epidemiology of dermatophyte infections. J Am Acad Dermatol 31: S21-S25.

7. AMEEN M (2010) Epidemiology of superficial fungal infections. Clin Dermatol 28: 197-201.

8. ASTICCIOLI S, DI SILVERIO A, SACCO L, FUSI I, VINCENTIL, et al. (2008) Dermatophyte infections in patients attending a tertiary care hospital in northernItaly. New Microbiol 31(4): 543-548.

9. DRAKENSJÖ IT, CHRYSSANTHOU E (2011) Epidemiology of dermatophyte infections in Stockholm, Sweden:a retrospective study from 2005-2009. Med Mycol 49: 484-488.

10. HAVLICKOVA B, CZAIKA VA, FRIEDRICH M (2008) Epidemiological trends in skin mycoses worldwide. Mycoses 51 (Suppl. 4): 2-15.

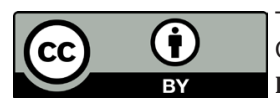

This work is licensed under Creative Commons Attribution 4.0 License

DOI: 10.19080/JPCR.2017.03.555602

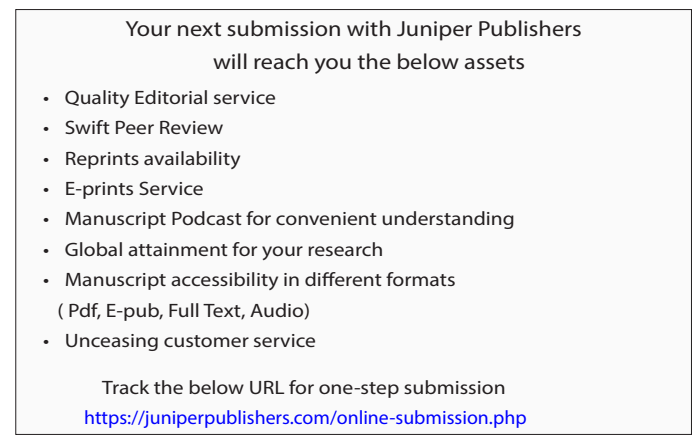

